\title{
Review of: "LincRNA"
}

Andrés Lanzós

Potential competing interests: The author(s) declared that no potential competing interests exist.

Just add what LincRNA stands for: Long intervening non coding RNA 